doi:10.13108/2020-12-2-10

\title{
OVERDETERMINED NEUMANN BOUNDARY VALUE PROBLEM IN UNBOUNDED DOMAINS
}

\author{
V.V. VOLCHKOV, VIT.V. VOLCHKOV
}

\begin{abstract}
The studying of overdetermined boundary value problems for elliptic partial differential equations was initiated by J. Serrin in 1971. In his work, he established a property of radial symmetry for solutions of some overdetermined Poisson problem. Apart of a significant independent interest, the problems of such kind have important applications in the potential theory, integral geometry, hydrodynamics and capillarity theory. Usually, the resolving of these problems is based on Hopf lemma on an angular boundary point and the method of hyperplanes motion introduced by A.A. Alexandrov for studying some geometric problems related with characterizing the spheres. Among other more modern methods not involving the maximum principle for the considered problems we mention the duality method, the method of volume derivative as well as an integral method.

In the present paper we consider an overdetermined Neumann problem for the Laplace equation $\Delta f=0$ in planar unbounded domains. We show that under some conditions, see Theorem 1 in Section 1, such problem is solvable only for the exterior of a ball. A specific feature of Theorem 1 is that in this theorem, for the first time, we obtain an exact condition for the growth of $f$ at infinity. Moreover, as Theorem 2 in Section 2 shows, other conditions in Theorem 1 are also necessary. In contrast to the earlier works, the proof of Theorem 1 employs some boundary properties of conformal mappings, Smirnov theorem on functions in a class $H_{p}$ and Fejer-Riesz theorem on non-negative trigonometrical polynomials.
\end{abstract}

Keywords: overdetermined problems, Neumann problem, harmonic functions, boundary behavior.

Mathematics Subject Classification: 31A05

\section{INTRODUCTION}

In 1971, J. Serrin initiated [1] the studying of overdetermined boundary value problems for elliptic partial differential equations. Later it was found that apart of a significant independent interest, such problems have important applications in the potential theory [2], integral geometry [3], 4], hydrodynamics [5], electrostatics [6], [7], and capillarity theory [8], 9]. For instance, a known Pompeiu problem still not completely resolved is reduced to an overdetermined boundary value problem for the Helmholtz equation [4, [10], [11] under rather general assumptions. Moreover, exactly the studying of such problems produced the deepest results on the regularity of boundaries of sets considered in the Pompeiu problem [3], [12], [13]. The results on an old Schiffer conjecture related to the discussed issues can be found by an interesting reader in [14], [15], see also the references therein.

A classical result by J. Serrin [1] establishes the property of a radial symmetry for the solutions of the following overdetemined Poisson problem.

V.V. Volchkov, Vit.V. Volchkov, Overdetermined Neumann Boundary Value Problem in unBOUNDED DOMAINS.

(c) Volchkov V.V., Volchkov Vit.V. 2020.

Submitted October 30, 2019. 
Let $\Omega$ be a bounded domain in $\mathbb{R}^{n}, n \geqslant 2$, with a boundary $\partial \Omega$ in the class $C^{2}$, for which there exists a function $f \in C^{2}(\bar{\Omega})$ solving the Poisson equation

$$
\Delta f=-1 \quad \text { in } \Omega
$$

and the boundary conditions

$$
f=0, \quad \frac{\partial f}{\partial n}=\text { const } \quad \text { on } \quad \partial \Omega .
$$

Then, as it was shown in [1, $\Omega$ is a ball and $f$ is radially symmetric. It was also mentioned in [1] that such statement remains true for some other equations generalizing (1.1) if in addition to $(1.2)$, the following condition is satisfied

$$
f>0 \text { in } \Omega \text {. }
$$

Serrin theorem was further developed and specified in various directions, see survey [16] with a wide list of references. First, in a series of works, there were specified assumptions of this theorem related with the smoothness of the boundary of $\Omega$ and of the right hand side $f$. Second, similar problems were considered for other equations including nonlinear ones and for other boundary conditions. Third, analogues of Serrin theorem were studied for other types of domains like perforated ones, annuli, unbounded domains. At that, in the case of an unbounded domain, in addition to conditions (1.2), (1.3), a condition on the behavior of the function $f$ at infinity was imposed. For instance, in work [17] it was assumed that $f$ tends to zero at infinity, while in [8], [18] it was additionally supposed that the same condition was holds for all first derivatives of $f$.

The proof of the main results in the most of the cited works was based on the maximum principle, Hopf lemma on an angular point and the method of hyperplanes motion introduced by A.D. Alexandrov for resolving some geometric problems related with characterization of spheres, see [16], [19], [20]. Among other, more modern methods not involving the maximum principle, we mention the duality method [21, the volume derivative method [22], and the integral method [23].

In the present paper we consider an overdetermined Neumann problem for the Laplace equation $\Delta f=0$ in planar unbounded domains. We show that under certain conditions, see Theorem 2.1 in Section 1 such problem is solvable only in the exterior of a circle. A specific feature of Theorem 2.1 is that an exact condition for the growth of $f$ at infinity is obtained. Moreover, as we see in Theorem 2.2 in Section 2, other conditions in Theorem 2.1 are also necessary. In contrast to the works of the predecessors, the proof of Theorem 2.1 employs some boundary properties of the conformal mappings, Smirnov theorem on the functions in the class $H_{p}$ and Fejer-Riesz theorem on non-negative trigonometric polynomials.

\section{Formulation of main Results}

Let $\Gamma$ be a closed smooth Jordan curve in a complex plane $\mathbb{C}$ and $G$ be a bounded domain $\mathbb{C}$ with the boundary $\Gamma, \bar{G}=G \cup \Gamma$. As usually, the symbol $\frac{\partial}{\partial n}$ stands for the operator of differentiating along the outward to $G$ normal to $\Gamma$.

Our main results as follows.

Theorem 2.1. Assume that there exists a function $f$ continuous in $\mathbb{C} \backslash G$ and harmonic in $\mathbb{C} \backslash \bar{G}$ and satisfying the following conditions:

1. $f=0$ on $\Gamma$;

2. $\frac{\partial f}{\partial n}=1$ on $\Gamma$;

3. $f(z)=o\left(|z|^{2}\right)$ as $z \rightarrow \infty$. 
Then the domain $G$ is a circle and

$$
f(z)=R \ln \frac{\left|z-z_{0}\right|}{R},
$$

where $z_{0}, R$ is the center and the radius of the circle $G$.

The proof of Theorem 2.1 is provided in Section 3. It is based on applying a conformal mapping of the exterior of the unit circle onto the domain $\mathbb{C} \backslash \bar{G}$. This mapping allows us to reduce the original problem in the domain $\mathbb{C} \backslash \bar{G}$ to an overdetermined boundary value problem, in which the main difficulty is an inhomogeneous boundary condition for the normal derivative. In order to study this condition, we need some gentle results on boundary properties of the function making the aforementioned conformal mapping as well as some properties of the Hardy classes $H_{p}$ in the unit circle, see [24, Chs. 9, 10]. Auxiliary constructions and statements are given in Section 2. We note that the absence of a similar theory of conformal mappings in a multi-dimensional case leave open an issue on analogue of Theorem 2.1 in the space $\mathbb{R}^{n}$ as $n>2$.

The following result shows the necessity of Conditions 1,2 and the sharpness of Condition 3 in Theorem 2.1.

Theorem 2.2. There exist a bounded domain $G \subset \mathbb{C}$ with a Jordan boundary $\Gamma$ of the class $C^{\infty}$ not coinciding with a circle and functions $f_{1}, f_{2}, f_{3}$ belonging to $C^{\infty}(\mathbb{C} \backslash G)$ and harmonic in $\mathbb{C} \backslash \bar{G}$ such that

1. $f_{1}$ satisfies Conditions 1 and 3 of Theorem 2.1;

2. $f_{2}$ satisfies Conditions 2 and 3 of Theorem 2.1;

3. $f_{3}$ satisfies Conditions 1, 2 of Theorem 2.1 and at that,

$$
f_{3}(z)=O\left(|z|^{2}\right) \quad \text { as } z \rightarrow \infty \text {. }
$$

The proof of Theorem 2.2 is provided in Section 4.

\section{Auxiliary constructions}

Let $A=\{z \in \mathbb{C}:|z|>1\}$ and a function $u$ be continuous in $\bar{A}=\{z \in \mathbb{C}:|z| \geqslant 1\}$. For each fixed $\rho \geqslant 1$, the Fourier series of the function $u\left(\rho e^{i \varphi}\right)$ reads as

$$
u\left(\rho e^{i \varphi}\right)=\sum_{n=-\infty}^{\infty} u_{n}(\rho) e^{i n \varphi}, \quad \varphi \in[0,2 \pi]
$$

where

$$
u_{n}(\rho)=\frac{1}{2 \pi} \int_{0}^{2 \pi} u\left(\rho e^{i \varphi}\right) e^{-i n \varphi} d \varphi
$$

It follows from 3.2 that

$$
\left|u_{n}(\rho)\right| \leqslant \max _{\varphi \in[0,2 \pi]}\left|u\left(\rho e^{i \varphi}\right)\right|
$$

for all $n \in \mathbb{Z}, \rho \geqslant 1$. Let $\Delta=\frac{\partial^{2}}{\partial x^{2}}+\frac{\partial^{2}}{\partial y^{2}}$ be the Laplace operator. If $u \in C^{2}(A)$, simple calculations and (3.2) show that for all $\rho>1, \varphi \in[0,2 \pi], n \in \mathbb{Z}$ the identity

$$
\Delta\left(u_{n}(\rho) e^{i n \varphi}\right)=\frac{1}{2 \pi} \int_{0}^{2 \pi}(\Delta u)\left(\rho e^{i t}\right) e^{i n(\varphi-t)} d t
$$

holds true. Moreover,

$$
\Delta\left(u_{n}(\rho) e^{i n \varphi}\right)=\left(u_{n}^{\prime \prime}(\rho)+\frac{u_{n}^{\prime}(\rho)}{\rho}-\frac{n^{2}}{\rho^{2}} u_{n}(\rho)\right) e^{i n \varphi} .
$$


By Riemann theorem on conformal mapping there exists a unique holomorphic in the domain $A$ function $w=\psi(z)$ mapping conformally $A$ onto the domain $\mathbb{C} \backslash G$ under the conditions

$$
\psi(\infty)=\infty, \quad \psi^{\prime}(\infty)=a>0
$$

The former condition in (3.6) shows that the function $w=\psi(z)$ maps the point $z=\infty$ into the point $w=\infty$, while the second condition means that

$$
\lim _{z \rightarrow \infty} \frac{\psi(z)}{z}=a>0
$$

It follows from Conditions (3.6) and (3.7) that the function $\psi$ being holomorphic in the domain $A$ has a simple pole at the point $z=\infty$ and this is why its Laurent series in $A$ reads as

$$
\psi(z)=a z+\sum_{0}^{\infty} \psi_{n} z^{-n}, \quad a>0 .
$$

According the principle of boundaries correspondence under conformal mappings, the function $\psi$ can be continuously continued on the set $\bar{A}$. For this continuation we keep the same notation $w=\psi(z)$.

Let $\mathbb{D}=\{z \in \mathbb{C}:|z|<1\}, \mathbb{T}=\{z \in \mathbb{C}:|z|=1\}$. As usually, we denote by $H_{p}(\mathbb{D}), p>0$, the class of functions $f$ holomorphic in $\mathbb{D}$ such that the integral

$$
\int_{0}^{2 \pi}\left|f\left(r e^{i \varphi}\right)\right|^{p} d \varphi
$$

is bounded as $0 \leqslant r<1$. For $z \in \mathbb{D}$ we let

$$
h(z)=a-\sum_{0}^{\infty} n \psi_{n} z^{-n-1}=\psi^{\prime}\left(\frac{1}{z}\right) .
$$

We note that since $\psi$ is univalent, the function $h$ has no zeroes in $\mathbb{D}$. In what follows we shall make use of some auxiliary statements on the properties of the functions $h$ and $\psi$.

Lemma 3.1. The functions $h$ and $1 / h$ belong to the class $H_{p}(\mathbb{D})$ for all $p>0$.

Proof. Let $b \in G$. We denote by $d(b)$ the distance from the point $b$ to $\Gamma$. Then for each $z \in \mathbb{D}$ we have

$$
\left|\psi\left(\frac{1}{z}\right)-b\right| \geqslant d(b)>0 .
$$

It follows from this inequality and 3.8 that there exists a constant $c_{1}>0$ such that

$$
\left|z\left(\psi\left(\frac{1}{z}\right)-b\right)\right|>c_{1}
$$

for all $z \in \mathbb{D}$. Moreover, by (3.8) and the definition of $\psi$ we obtain that

$$
\left|z\left(\psi\left(\frac{1}{z}\right)-b\right)\right|<c_{2}, \quad z \in \mathbb{D},
$$

where $c_{2}>0$ is independent of $z$. For $z \in \mathbb{D}$, by 3.10 we find:

$$
h(z)=-z^{2}\left(\psi\left(\frac{1}{z}\right)-b\right)^{2} \lambda^{\prime}(z),
$$

where

$$
\lambda(z)=\left(\psi\left(\frac{1}{z}\right)-b\right)^{-1}
$$


Taking into consideration estimates (3.11) and (3.12), for all $p>0, r \in[0,1)$ by (3.13) we obtain:

$$
\int_{0}^{2 \pi}\left(\left|h\left(r e^{i \varphi}\right)\right|^{p}+\left|h\left(r e^{i \varphi}\right)\right|^{-p}\right) d \varphi<c_{2}^{2 p} \int_{0}^{2 \pi}\left|\lambda^{\prime}\left(r e^{i \varphi}\right)\right|^{p} d \varphi+c_{1}^{-2 p} \int_{0}^{2 \pi}\left|\lambda^{\prime}\left(r e^{i \varphi}\right)\right|^{-p} d \varphi .
$$

The function $\lambda$ maps the circle $\mathbb{D}$ conformally and univalently onto some bounded domain with a smooth Jordan boundary. By the Lindelöf theorem, see [24, Ch. 10, Sect. 1, Thm. 4], under an appropriate choice, the branches of the argument of the function $\arg \lambda^{\prime}(z)$ and $\arg \frac{1}{\lambda^{\prime}(z)}$ can be continued to continuous functions on $\overline{\mathbb{D}}$. This implies [24, Ch. 10, Sect. 1, Thm. 5] that the functions $\lambda$ and $1 / \lambda$ belong to the class $H_{p}(\mathbb{D})$ for each $p>0$. Thus, the integrals in the right hand side of inequality (3.14) are bounded in $r$. Together with (3.14 this completes the proof.

Corollary 3.1. Almost everywhere in $\mathbb{T}$, the function $h$ possesses finite limiting values over non-tangential paths; these values form a boundary function $h\left(e^{i \varphi}\right), \varphi \in(0,2 \pi)$. At that, $h\left(e^{i \varphi}\right) \in L^{p}(0,2 \pi)$ for each $p>0$ and

$$
h\left(e^{i \varphi}\right) \neq 0 \text { for almost all } \varphi \in(0,2 \pi) .
$$

Proof. The existence of the mentioned limiting function $h\left(e^{i \varphi}\right)$ and its belonging to the class $L^{p}(0,2 \pi)$ is implied by Lemma 3.1 and by a well-known property for a each function in the class $H_{p}(\mathbb{D})$, see, for instance, 24 , Ch. 9, Sect. 4]. Applying this property to the function $1 / h$ and employing Lemma 3.1, we obtain 3.15.

Corollary 3.2. Let $\alpha, \beta \in \mathbb{C}$ and $|\alpha| \geqslant|\beta|>0$. Then the function

$$
h_{1}(z)=\frac{h(z)}{(\alpha+\beta z)^{2}}
$$

belongs to the class $H_{p}(\mathbb{D})$ for all $0<p<\frac{1}{4}$.

Proof. We first assume that $|\beta|=|\alpha|$. In this case $\alpha+\beta z=\beta\left(z-e^{i \gamma}\right)$ for some $\gamma \in[0,2 \pi]$. Let $0<p<\frac{1}{4}$. We choose $q>1$ so that $q p<\frac{1}{4}$. Employing Hölder inequality and Lemma 3.1 . for each $r \in[0,1)$ we have:

$$
\begin{aligned}
\int_{0}^{2 \pi}\left|h_{1}\left(r e^{i \varphi}\right)\right|^{p} d \varphi & =\int_{0}^{2 \pi}\left|h\left(r e^{i \varphi}\right)\right|^{p}\left|\beta\left(r e^{i \varphi}-e^{i \gamma}\right)\right|^{-2 p} d \varphi \\
& \leqslant\left(\int_{0}^{2 \pi}\left|h\left(r e^{i \varphi}\right)\right|^{\frac{p q}{q-1}} d \varphi\right)^{\frac{q-1}{q}}\left(\int_{0}^{2 \pi}\left|\beta\left(r e^{i \varphi}-e^{i \gamma}\right)\right|^{-2 p q} d \varphi\right)^{\frac{1}{q}} \\
& \leqslant c\left(\int_{0}^{2 \pi}\left|1-r e^{i(\varphi-\gamma)}\right|^{-2 p q} d \varphi\right)^{\frac{1}{q}},
\end{aligned}
$$

where a constant $c>0$ is independent of $r$. Since

$$
\left|1-r e^{i(\varphi-\gamma)}\right| \geqslant 1-\cos (\varphi-\gamma)
$$

and $p q<\frac{1}{4}$, by $(3.16)$ we obtain a desired statement. In the case $|\alpha|>|\beta|>0$ the statement is obvious in view of Lemma 3.1 and the inequality

$$
|\alpha+\beta z| \geqslant|\alpha|-|\beta|, \quad z \in \mathbb{D} \text {. }
$$

The proof is complete.

Lemma 3.2. For almost each $\varphi \in[0,2 \pi]$ in sense of the Lebesgue measure, the identity

$$
\lim _{\varepsilon \rightarrow+0} \psi^{\prime}\left((1+\varepsilon) e^{i \varphi}\right)=\lim _{\varepsilon \rightarrow+0} \frac{\psi\left((1+\varepsilon) e^{i \varphi}\right)-\psi\left(e^{i \varphi}\right)}{\varepsilon e^{i \varphi}}
$$

holds, where both limits exist and are finite. 
Proof. By relation (3.10) we have

$$
\psi^{\prime}\left((1+\varepsilon) e^{i \varphi}\right)=h\left((1+\varepsilon)^{-1} e^{-i \varphi}\right)
$$

for all $\varepsilon>0, \varphi \in[0,2 \pi]$. Together with Corollary 3.1 this implies the existence of a finite limit in the left hand side of identity (3.17) for almost each $\varphi \in[0,2 \pi]$. By the mean theorem, for such $\varphi$, the identity holds:

$$
\int_{1}^{1+\varepsilon} \psi^{\prime}\left(\rho e^{i \varphi}\right) d \rho=\varepsilon \psi^{\prime}\left(\xi e^{i \varphi}\right)
$$

for each $\varepsilon>0$ and some $\xi \in(1,1+\varepsilon)$ independent of $\varepsilon$. Therefore, there exists a finite limit

$$
\lim _{\varepsilon \rightarrow+0} \frac{1}{\varepsilon} \int_{1}^{1+\varepsilon} \psi^{\prime}\left(\rho e^{i \varphi}\right) d \rho
$$

which is equal to the limit in the left hand side in identity (3.17). Since

$$
\int_{1}^{1+\varepsilon} \psi^{\prime}\left(\rho e^{i \varphi}\right) d \rho=\frac{\psi\left((1+\varepsilon) e^{i \varphi}\right)-\psi\left(e^{i \varphi}\right)}{e^{i \varphi}},
$$

this implies the statement of Lemma 3.2. The proof is complete.

Lemma 3.3. For almost each $\varphi \in(0,2 \pi)$, there exists $\delta=\delta(\varphi)>0$ such that as $\varepsilon \in$ $(0, \delta(\varphi))$, the circle

$$
K_{\varepsilon, \varphi}=\left\{z \in \mathbb{C}:\left|z-\psi\left(e^{i \varphi}\right)-\varepsilon e^{i \varphi} \psi^{\prime}\left(e^{i \varphi}\right)\right| \leqslant \frac{\varepsilon}{2}\left|\psi^{\prime}\left(e^{i \varphi}\right)\right|\right\}
$$

is disjoint with $\Gamma$.

Proof. We assume that for some $\varphi \in(0,2 \pi)$ there exists a sequence $\left\{\varepsilon_{j}\right\}_{j=1}^{\infty}$ of positive numbers such that $\lim _{j \rightarrow \infty} \varepsilon_{j}=0$ and for each $j$ the circle $K_{\varepsilon_{j}, \varphi}$ intersects $\Gamma$. We denote by $\varphi_{j}$ one of the points in the semi-interval $[0,2 \pi)$, for which $\psi\left(e^{i \varphi_{j}}\right) \in K_{\varepsilon_{j}, \varphi} \cap \Gamma$. Then we have

$$
\left|\psi\left(e^{i \varphi_{j}}\right)-\psi\left(e^{i \varphi}\right)-\varepsilon_{j} e^{i \varphi} \psi^{\prime}\left(e^{i \varphi}\right)\right| \leqslant \frac{\varepsilon_{j}}{2}\left|\psi^{\prime}\left(e^{i \varphi}\right)\right| .
$$

By this inequality and the univalentness of $\psi$ we conclude that $\varphi_{j} \rightarrow \varphi$ as $j \rightarrow \infty$.

The function $\psi\left(e^{i t}\right)$ is absolutely continuous on $[0,2 \pi]$ and for almost all $t \in[0,2 \pi]$ the identity

$$
\frac{d}{d t} \psi\left(e^{i t}\right)=i e^{i t} \psi^{\prime}\left(e^{i t}\right)
$$

holds true, see [24, Ch. 10, Sect. 1, Thm. 1].

Assume now that $\psi^{\prime}\left(e^{i \varphi}\right) \neq 0$ and

$$
\psi\left(e^{i \varphi}\right)-\psi\left(e^{i \varphi_{j}}\right)=i \psi^{\prime}\left(e^{i \varphi}\right) e^{i \varphi}\left(\varphi-\varphi_{j}\right)+o\left(\varphi-\varphi_{j}\right) \quad \text { as } \quad j \rightarrow \infty .
$$

It follows from Corollary 3.1 and identity $(3.20)$ that these conditions are satisfied for almost all $\varphi \in(0,2 \pi)$. Comparing (3.21) and (3.19) and bearing in mind that $\psi^{\prime}\left(e^{i \varphi}\right) \neq 0$, we arrive at the inequality

$$
\left|\varepsilon_{j}+i\left(\varphi-\varphi_{j}\right)+o\left(\varphi-\varphi_{j}\right)\right| \leqslant \frac{\varepsilon_{j}}{2} \quad \text { as } \quad j \rightarrow \infty .
$$

For sufficiently large $j$ the latter inequality is contradictory and this completes the proof.

Lemma 3.4. Let a function $f$ be harmonic in the circle $K=\left\{\zeta \in \mathbb{C}:\left|\zeta-\zeta_{0}\right|<r\right\}$ and

$$
M=\sup _{\zeta \in K}|f(\zeta)|<+\infty .
$$

Then for each $\zeta \in K$ the estimate

$$
\left|f(\zeta)-f\left(\zeta_{0}\right)\right| \leqslant \frac{2 M\left|\zeta-\zeta_{0}\right|}{r-\left|\zeta-\zeta_{0}\right|}
$$

holds. 
Proof. The assumptions of the lemma imply that the function $w(z)=f\left(r z+\zeta_{0}\right)$ is harmonic in $\mathbb{D}$ and $|w(z)| \leqslant M$ for each $z \in \mathbb{D}$. This implies, see, for instance, [24, Ch. 9, Sect. 2, Cor. 2 ] that almost everywhere on $\mathbb{T}$ the function $w$ possesses non-tangential finite limiting values. As usually, we keep the notation $w\left(e^{i t}\right)$ for the corresponding limiting function defined almost everywhere on $\mathbb{T}$. Then by $(3.22)$ we get $\left|w\left(e^{i t}\right)\right| \leqslant M$. Moreover, for each $z=\rho e^{i \varphi}, 0 \leqslant \rho<1$, the Poisson formula holds:

$$
w(z)-w(0)=\frac{1}{\pi} \int_{0}^{2 \pi} w\left(e^{i t}\right) \sum_{n=1}^{\infty} \rho^{n} \cos (n(\varphi-t)) d t,
$$

see [24, Ch. 9, Sect. 2, Thm. 3]. The integral in the right hand side does not exceed the expression

$$
\frac{1}{\pi} \int_{0}^{2 \pi}\left|w\left(e^{i t}\right)\right| \sum_{n=1}^{\infty} \rho^{n} d t \leqslant \frac{2 M \rho}{1-\rho}
$$

and this is why estimate 3.23 follows 3.24$)$. The proof is complete.

Lemma 3.5. For almost each $\varphi \in(0,2 \pi)$, the following statement holds: if $f$ is harmonic in $\mathbb{C} \backslash \bar{G}$, continuous in $\mathbb{C} \backslash G$, and there exists a derivative $\frac{\partial f}{\partial n}\left(\psi\left(e^{i \varphi}\right)\right)$, then

$$
\lim _{\varepsilon \rightarrow+0} \frac{f\left(\psi\left((1+\varepsilon) e^{i \varphi}\right)\right)-f\left(\psi\left(e^{i \varphi}\right)\right)}{\varepsilon}=\frac{\partial f}{\partial n}\left(\psi\left(e^{i \varphi}\right)\right)\left|\psi^{\prime}\left(e^{i \varphi}\right)\right| .
$$

Proof. It follows from Corollary 3.1 and Lemma 3.2 that for almost each $\varphi \in(0,2 \pi)$ there exists a finite non-zero limit in the left hand side of identity (3.17) and

$$
\psi\left((1+\varepsilon) e^{i \varphi}\right)=\psi\left(e^{i \varphi}\right)+\varepsilon e^{i \varphi} \psi^{\prime}\left(e^{i \varphi}\right)+o(\varepsilon) \quad \text { as } \quad \varepsilon \rightarrow+0 .
$$

Therefore, for such $\varphi$, the point $\psi\left((1+\varepsilon) e^{i \varphi}\right)$ is in the circle $K_{\varepsilon, \varphi}$, see 3.18$)$, for all sufficiently small $\varepsilon>0$. We apply Lemma 3.4 letting

$$
\zeta_{0}=\psi\left(e^{i \varphi}\right)+\varepsilon e^{i \varphi} \psi^{\prime}\left(e^{i \varphi}\right), \quad r=\frac{\varepsilon}{2}\left|\psi^{\prime}\left(e^{i \varphi}\right)\right|, \quad \zeta=\psi\left((1+\varepsilon) e^{i \varphi}\right),
$$

where $\varepsilon \in(0, \delta(\varphi))$ is small enough. Since the circle $K_{\varepsilon, \varphi}$ is contained in the circle

$$
\left\{z \in \mathbb{C}:|z| \leqslant\left|\psi\left(e^{i \varphi}\right)\right|+\frac{3}{2} \delta(\varphi)\left|\psi^{\prime}\left(e^{i \varphi}\right)\right|\right\}
$$

there exists a constant $M_{\varphi}$ independent of $\varepsilon$ such that

$$
\sup _{K_{\varepsilon, \varphi}}|f| \leqslant M_{\varphi} \quad \text { for all } \quad \varepsilon \in(0, \delta(\varphi))
$$

Then by 3.25 and Lemma 3.4 we obtain:

$$
f\left(\psi\left((1+\varepsilon) e^{i \varphi}\right)\right)-f\left(\psi\left(e^{i \varphi}\right)+\varepsilon e^{i \varphi} \psi^{\prime}\left(e^{i \varphi}\right)\right)=o(\varepsilon) \quad \text { as } \quad \varepsilon \rightarrow+0 .
$$

Then, by the definition of the normal derivative we find that

$$
\frac{\partial f}{\partial n}\left(\psi\left(e^{i \varphi}\right)\right)=\lim _{\varepsilon \rightarrow+0} \frac{f\left(\psi\left(e^{i \varphi}\right)+\varepsilon e^{i \varphi} \psi^{\prime}\left(e^{i \varphi}\right)\right)-f\left(\psi\left(e^{i \varphi}\right)\right)}{\varepsilon\left|\psi^{\prime}\left(e^{i \varphi}\right)\right|} .
$$

This identity and relation 3.26 imply the statement of the lemma and complete the proof. 


\section{Proof of TheOREM 1}

Let a function $f$ satisfies the assumptions of Theorem 1. We are going to prove that $G$ is a circle and

$$
f(z)=R \ln \frac{\left|z-z_{0}\right|}{R},
$$

where $z_{0}, R$ are the center and radius of the circle $G$.

We consider the function $u(z)=f(\psi(z))$, where $\psi$ is a function defined in Section 2. The assumptions of Theorem 1 imply that $u$ is harmonic in the domain $A$ and is continuous in $\bar{A}$, and at that,

$$
u\left(e^{i \varphi}\right)=0 \text { for each } \varphi \in[0,2 \pi] .
$$

Moreover, it follows from Condition 3 of Theorem 1 and identity (3.8) that

$$
u(z)=o\left(|z|^{2}\right) \quad \text { as } \quad z \rightarrow+\infty .
$$

For each fixed $\rho \geqslant 1$, the Fourier series of the function $u\left(\rho e^{i \varphi}\right)$ is given by (3.1), where $u_{n}(\rho)$ are continuous on $[1,+\infty)$, see (3.2). By the harmonicity of $u$ and relation (3.4) we obtain that the functions $u_{n}(\rho) e^{i n \varphi}$ are harmonic in $A$ for all $n$. By (3.5) this means that

$$
u_{0}(\rho)=a_{0}+b_{0} \ln \rho, \quad u_{n}(\rho)=a_{n} \rho^{n}+b_{n} \rho^{-n} \quad \text { as } \quad n \neq 0,
$$

where $\rho \geqslant 1$ and $a_{n}, b_{n}$ are complex constants. Identities 4.1 and $(3.2)$ imply that $u_{n}(1)=0$ and hence,

$$
a_{0}=0 \quad \text { and } \quad a_{n}+b_{n}=0 \text { as } n \neq 0 .
$$

By (3.3) and 4.2 we get

$$
u_{n}(\rho)=o\left(\rho^{2}\right) \quad \text { as } \quad \rho \rightarrow+\infty .
$$

The latter identity means that $a_{n}=0$ and $b_{-n}=0$ as $n \geqslant 2$. Comparing this with (4.3), we conclude that

$$
u(z)=b_{0} \ln |z|+a_{1}\left(z-\frac{1}{\bar{z}}\right)+a_{-1}\left(\frac{1}{z}-\bar{z}\right),
$$

as $|z| \geqslant 1$, where the bar denotes the complex conjugation. By this identity we find:

$$
\lim _{\varepsilon \rightarrow+0} \frac{u\left((1+\varepsilon) e^{i \varphi}\right)-u\left(e^{i \varphi}\right)}{\varepsilon}=b_{0}+2 a_{1} e^{i \varphi}-2 a_{-1} e^{-i \varphi}
$$

for each $\varphi \in[0,2 \pi]$. By Lemma 3.5 we then conclude that

$$
b_{0}+2 a_{1} e^{-i \varphi}-2 a_{-1} e^{i \varphi}=\left|h\left(e^{i \varphi}\right)\right|
$$

for almost each $\varphi \in[0,2 \pi]$.

By Fejer-Riesz theorem, see [25, App. 5], a non-negative trigonometric polynomial in the left hand side of identity 4.5 can be represented as

$$
b_{0}+2 a_{1} e^{-i \varphi}-2 a_{-1} e^{i \varphi}=\left|\alpha+\beta e^{i \varphi}\right|^{2}, \quad \varphi \in[0,2 \pi],
$$

where complex constants $\alpha, \beta$ are such that

$$
\alpha+\beta z \neq 0 \quad \text { as } \quad z \in \mathbb{D} .
$$

Let us prove that $\beta=0$. We suppose the contrary, then by (4.7) we have $|\alpha| \geqslant|\beta|>0$. Applying now Lemma 3.1 and Corollary 3.2, we conclude that the functions

$$
h_{1}(z)=\frac{h(z)}{(\alpha+\beta z)^{2}}, \quad h_{2}(z)=\frac{1}{h_{1}(z)}
$$

belong to the class $H_{p}(\mathbb{D})$ as $p \in(0,1 / 4)$. Moreover, it follows from 4.5) and 4.7) that

$$
\left|h_{1}\left(e^{i \varphi}\right)\right|=\left|h_{2}\left(e^{i \varphi}\right)\right|=1
$$


for almost each $\varphi \in(0,2 \pi)$. By Smirnov theorem, see [24, Ch. 9, Sect. 4, Thm. 4] this means that $\left|h_{1}(z)\right|=1$ for all $z \in \mathbb{D}$. Therefore,

$$
h(z)=\gamma\left(\alpha^{2}+2 \alpha \beta z+\beta^{2} z^{2}\right)
$$

where $\gamma \in \mathbb{C},|\gamma|=1$. Taking into consideration (3.10), we then obtain that $\beta=0$ and this contradicts our assumption. This arguing and formulae (4.5) and (4.6) show that $\beta=a_{1}=$ $a_{-1}=0$ and $\left|h\left(e^{i \varphi}\right)\right|=b_{0}$ for almost each $\varphi \in(0,2 \pi)$. As above, by Smirnov theorem we conclude that $h$ is identically constant. In view of $(3.10)$ and $(3.8)$, we get $h(z)=a$ in $\mathbb{D}$ and $\psi(z)=a z+\psi_{0}$ in $A$. Thus, letting $R=a$ and $z_{0}=\psi_{0}$, we arrive at the statement of Theorem 2.1,

\section{Proof of Theorem 2}

It is obvious that for all sufficiently small $\varepsilon \in(0,1)$, the inequality holds:

$$
\frac{2}{3}+\frac{1}{9(1-\varepsilon)^{2}}<(1-\varepsilon)^{2} .
$$

For such $\varepsilon$ we denote:

$$
A_{\varepsilon}=\{z \in \mathbb{C}:|z|>1-\varepsilon\}
$$

We consider a function

$$
\Phi(z)=z-\frac{2}{3 z}-\frac{1}{27 z^{3}}, \quad z \in A_{\varepsilon} .
$$

For all $z_{1}, z_{2} \in A_{\varepsilon}$ we have the estimates:

$$
\left|z_{1} z_{2}\right|>(1-\varepsilon)^{2}, \quad\left|z_{1}^{-2}+\left(z_{1} z_{2}\right)^{-1}+z_{2}^{-2}\right|<\frac{3}{(1-\varepsilon)^{2}}
$$

Moreover, by (5.2) we find:

$$
\Phi\left(z_{1}\right)-\Phi\left(z_{2}\right)=\left(z_{1}-z_{2}\right)\left(1+\frac{1}{z_{1} z_{2}}\left(\frac{2}{3}+\frac{1}{27}\left(z_{1}^{-2}+\left(z_{2} z_{2}\right)^{-1}+z_{2}^{-2}\right)\right)\right) .
$$

In view of inequalities (5.1) and (5.3), by the latter relation we conclude that $\Phi\left(z_{1}\right) \neq \Phi\left(z_{2}\right)$ as $z_{1} \neq z_{2}$. Thus, $\Phi$ is univalent in the domain $A_{\varepsilon}$. We let

$$
\Phi\left(A_{\varepsilon}\right)=\left\{z \in \mathbb{C}: z=\Phi(\zeta), \zeta \in A_{\varepsilon}\right\},
$$

and we denote by $g$ the inverse function for $\Phi$ acting from $\Phi\left(A_{\varepsilon}\right)$ on $A_{\varepsilon}$. It follows from formula (5.2) that for each $z \in A_{\varepsilon}$ the inequalities hold:

$$
|z|-\frac{2}{3(1-\varepsilon)}-\frac{1}{27(1-\varepsilon)^{3}}<|\Phi(z)|<|z|+\frac{2}{3(1-\varepsilon)}+\frac{1}{27(1-\varepsilon)^{3}} .
$$

By the definition of $g$ we obtain that

$$
|z|-\frac{2}{3(1-\varepsilon)}-\frac{1}{27(1-\varepsilon)^{3}}<|g(z)|<|z|+\frac{2}{3(1-\varepsilon)}+\frac{1}{27(1-\varepsilon)^{3}}
$$

for all $z \in \Phi\left(A_{\varepsilon}\right)$.

We let

$$
G=\mathbb{C} \backslash \overline{\Phi(A)}, \quad \text { where } \quad \Phi(A)=\{z \in \mathbb{C}: z=\Phi(\zeta), \zeta \in A\} .
$$

Since the function $\Phi$ is univalent, the set $G$ is a bounded domain with a smooth Jordan boundary $\Gamma=\{z \in \mathbb{C}: z=\Phi(\zeta), \zeta \in \mathbb{T}\}$. Moreover, since

the domain $G$ is not a circle.

$$
\Phi(1)=-\Phi(-1)=\frac{8}{27} \quad \text { and } \quad \Phi(i)=-\Phi(-i)=\frac{44}{27} i
$$


We consider the functions

$$
f_{1}=\ln |g|, \quad f_{2}=\frac{10}{9} \ln |g|-\frac{1}{3} \operatorname{Re}\left(\frac{1}{g^{2}}\right), \quad f_{3}=\frac{10}{9} \ln |g|+\frac{1}{6} \operatorname{Re}\left(g^{2}-\frac{1}{g^{2}}\right) .
$$

It follows from the definition $g$ that this function is holomorphic in $\Phi\left(A_{\varepsilon}\right)$ and is non-zero. This yields that the functions $f_{1}, f_{2}, f_{3}$ are harmonic in $\mathbb{C} \backslash \bar{G}$ and belong to the class $C^{\infty}(\mathbb{C} \backslash G)$. Employing also (5.4), we conclude that the functions $f_{1}, f_{2}, f_{3}$ satisfy all assumptions of Theorem 2.2 .

\section{BIBLIOGRAPHY}

1. J. Serrin. A symmetry problem in potential theory // Arch. Rat. Mech. Anal. 43:1, 304-318 (1971).

2. L.E. Payne, G.A. Philippin. On two free boundary problems in potential theory // J. Math. Anal. Appl. 161:2, 332-342 (1991).

3. C.A. Berenstein, M. Shahshahani. Harmonic analysis and the Pompeiu problem // Amer. J. Math. 105:5, 1217-1229 (1983).

4. V.V. Volchkov. Integral geometry and convolution equations. Kluwer, Dordrecht (2003).

5. R.L. Fosdick, J. Serrin. Rectilinear steady flow of simple fluids // Proc. R. Soc. Lond. A. 332:1590, 311-333 (1973).

6. G.A. Philippin. On a free boundary problem in Electrostatics // Math. Meth. Appl. Sci. 12:5, 387-392 (1990).

7. O. Mendez, W. Reichel. Electrostatic characterization of spheres // Forum Math. 12:2, 223-245 (2000).

8. B. Sirakov. Symmetry for exterior elliptic problems and two conjectures in potential theory // Ann. Inst. Henri Poincaré, Anal. non-Linéaire. 18:2, 135-156 (2001).

9. W. Reichel. Radial symmetry for an electrostatic, a capillarity and some fully nonlinear overdetermined problems on exterior domains // Z. Anal. Anwendungen. 15:3, 619-635 (1996).

10. L. Zalcman. A bibliographic survey of the Pompeiu problem // Approximation by solutions of partial differential equations (ed. Fuglede B. et. al), Kluwer, Dordrecht, 185-194 (1992).

11. V.V. Volchkov, Vit.V. Volchkov. Offbeat integral geometry on symmetric spaces. Birkhäuser, Basel (2013).

12. S.A. Williams. Analyticity of the boundary for Lipschitz domains without the Pompeiu property // Indiana Univ. Math. J. 30:3, 357-369 (1981).

13. L.A. Caffarelli, L. Karp, H. Shahgholian. Regularity of a free boundary with application to the Pompeiu problem // Ann. Math. 151:2, 269-292 (2000).

14. C.A. Berenstein, P. Yang. An overdetermined Neumann problem in the unit disk // Adv. Math. 44:1, 1-17 (1982).

15. N.B. Willms, G.M.L. Gladwell. Saddle points and overdetermined problems for the Helmholtz equation // Z. Angew Math. Phys. 45:1, 1-26 (1994).

16. P.W. Schaefer. On nonstandard overdetermined boundary value problems // Nonlinear Analysis. 47:4, 2203-2212 (2001).

17. N. Garofalo, E. Sartori. Symmetry in exterior boundary value problems for quasilinear elliptic equations via blow-up and a priori estimates // Adv. Diff. Eqs. 4:2, 137-161 (1999).

18. W. Reichel. Radial symmetry for elliptic boundary-value problems on exterior domains // Arch. Rat. Mech. Anal. 137:6, 381-394 (1997).

19. G.A. Philippin. Applications of the maximum principle to a variety of problems involving elliptic differential equations // Pitman Res. Notes Math. Ser. 175, 34-48 (1988).

20. A.D. Alexandrov. A characteristic property of the spheres // Ann. Mat. Pura Appl. 58:1, 303-315 (1962).

21. L.E. Payne, P.W. Schaefer. Duality theorems in some overdetermined problems // Math. Meth. Appl. Sciences. 11:6, 805-819 (1989).

22. M. Choulli, A. Henrot. Use of the domain derivative to prove symmetry results in partial differential equations // Math. Nachr. 192:1, 91-103 (1998). 
23. B. Brandolini, C. Nitsch, P. Salani, C. Trombetti. Serrin type overdetermined problems: an alternative proof // Arch. Rational Mech. Anal. 190:2, 267-280 (2008).

24. G.M. Goluzin. Geometric theory of functions of a complex variable. Nauka, Moscow (1966). [Transl. Math. Monog. 26. Amer. Math. Soc., Providence, RI (1969).]

25. B.Ya. Levin. Distribution of zeros of entire functions. Gostekhizdat, Moscow (1956). [Amer. Math. Soc., Providence, RI (1980).]

Valerii Vladimirovich Volchkov,

Donetsk National University,

Universitetskaya str. 24,

83001, Donetsk

E-mail: valeriyvolchkov@gmail.com

Vitalii Vladimirovich Volchkov,

Donetsk National University,

Universitetskaya str. 24,

83001, Donetsk

E-mail: volna936@gmail.com 